\title{
Effect of scene complexity on colour constancy with real three-dimensional scenes and objects
}

\author{
Sérgio M C Nascimento \\ Department of Physics, Gualtar Campus, University of Minho, 4710-057 Braga, Portugal; \\ e-mail: smcn@fisica.uminho.pt
}

Vasco M N de Almeida, Paulo T Fiadeiro

Remote Sensing Unit, Department of Physics, University of Beira Interior, 6201-001 Covilhã, Portugal

David H Foster

Computational Neuroscience Group, Faculty of Life Sciences, Moffat Building, University of Manchester, Manchester M60 1QD, UK

Received 3 December 2003, in revised form 5 November 2004

\begin{abstract}
The effect of scene complexity on colour constancy was tested with a novel technique in which a virtual image of a real 3-D test object was projected into a real 3-D scene. Observers made discriminations between illuminant and material changes in simple and complex scenes. The extent of colour constancy achieved varied little with either scene structure or test-object colour, suggesting a dominant role of local cues in determining surface-colour judgments.
\end{abstract}

\section{Introduction}

Despite large variations in the colour of the illuminant in a scene, the colours of the objects seem to change little. This colour constancy has, however, proved difficult to quantify experimentally (Foster 2003). A variety of methodologies have been employed, including colour naming (Troost 1992), asymmetric colour matching (Arend and Reeves 1986; Lucassen 1993), and achromatic adjustment (Brainard 1998; Kraft and Brainard 1999). The stimuli used in these kinds of experiments have typically been either computergenerated on monitor screens (Arend and Reeves 1986; Foster et al 2001) or real objects (Kraft and Brainard 1999) but in limited experimental designs. The main constraints have been low luminance levels, restricted colour gamut, the two-dimensionality of scenes or test objects, and the often abstract nature of the stimuli. Some or all of these factors have been invoked in explanations of the variable levels of colour constancy recorded.

Our aim here has been to test colour constancy without these constraints. A novel optical technique (de Almeida et al 2002; Nascimento et al 2004) was used to project a virtual image of a real 3-D test object into a real 3-D scene. The effect of chromatic complexity was tested by varying the number and colours of the real objects in the scene. Surprisingly, no systematic effect of scene complexity or test-object colour was detected.

\section{Methods}

\subsection{Apparatus}

Figure 1a shows the optical arrangement. The virtual image (V) of an illuminated 3-D test object $(\mathrm{O})$ was projected by a large 50/50 beam splitter (BS) into a 3-D scene. The beam splitter was a $400 \mathrm{~mm} \times 300 \mathrm{~mm}$ borosilicate glass plate, $1 \mathrm{~mm}$ thick, with a Melles Griot HEBBAR coating. No secondary reflections from the back surface were visible. The scene was illuminated by a computer-driven LCD data projector (Epson EMP-5600; 2200 ANSI lumens) and the test object was illuminated independently by another LCD data projector (Sony VPL-CS10; 1000 ANSI lumens) driven by a graphics card VSG2/5 (Cambridge Research Systems, UK) with 12-bit resolution in each of the R, $\mathrm{G}$, and $\mathrm{B}$ channels. The test object was always a cube and was seen by observers as part of the scene and not distinguished by virtue of its origin, even under moderate 
head movements. Mutual reflections between the test object and nearby surfaces were simulated by introducing appropriately coloured surfaces close to O. These 'auxiliary' surfaces had the same colours as the surfaces located in the scene near the virtual image $\mathrm{V}$ and could not be seen by the observer.

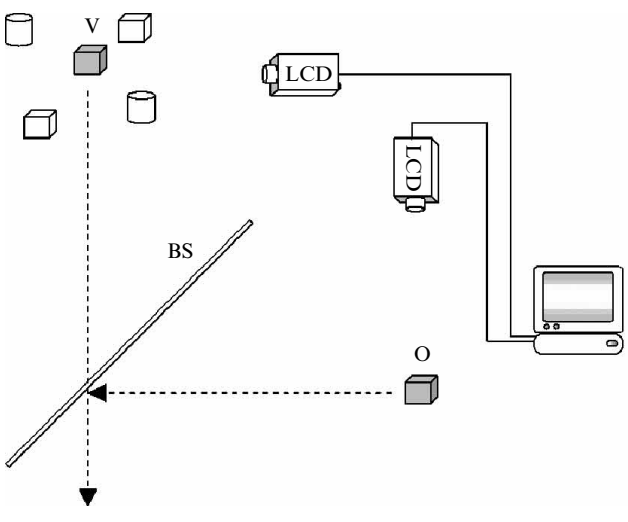

(a)

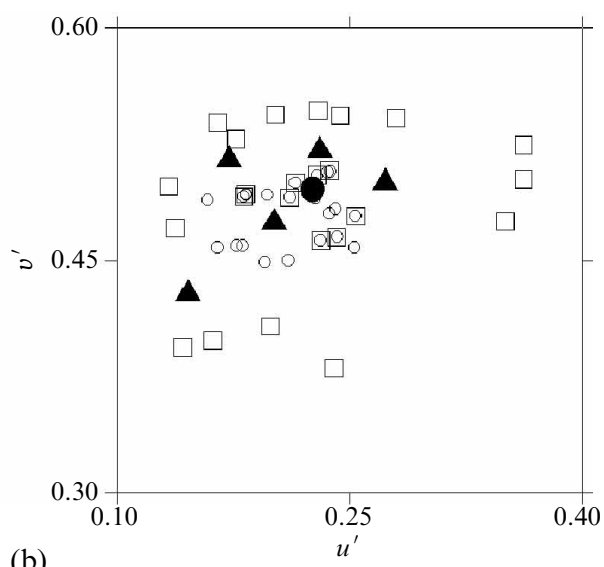

Figure 1. (a) Optical apparatus. The observer viewed a real scene comprising 24 real 3-D objects through the large beam splitter (BS), which projected into the scene a virtual image (V) of a cube test object $(\mathrm{O})$. The scene and test object were illuminated by independent computer-driven LCD projectors. (b) Coordinates in the CIE $1976\left(u^{\prime}, v^{\prime}\right)$ space of the coloured test objects used in the scenes when illuminated under CIE $\mathrm{D}_{65}$. Solid circle: uniform surround of scene 1; open circles: objects from unsaturated scene (scene 2); open squares: objects from saturated scene (scene 3); triangles: test objects.

\subsection{Scenes}

Three types of scenes were tested. Scene 1 had a simple uniform surround; scene 2 had a complex spatial surround of 24 objects of different shapes and of unsaturated colours; and scene 3 had a complex surround of the same objects as in scene 2 but some with more saturated colours. Figure $1 \mathrm{~b}$ represents in the CIE $1976\left(u^{\prime}, v^{\prime}\right)$ colour space the coordinates of the objects in the scenes when illuminated under CIE standard illuminant $\mathrm{D}_{65}$. The colours of the test objects, also represented in figure $1 \mathrm{~b}$, were variously red, green, blue, yellow, and white, and were distinct from the other colours in the scene. The complete scene subtended $10 \mathrm{deg}$ visual angle and the test object $1.5 \mathrm{deg}$. The background was a dark-grey wall, dimly illuminated by stray light from the scene and data projector. The total visual field was about $30 \mathrm{deg}$. The illumination on the scene from the LCD data projector had a correlated colour temperature of either $25000 \mathrm{~K}$ or $6700 \mathrm{~K}$ and luminance of about $400 \mathrm{~cd} \mathrm{~m}^{-2}$ measured at a barium sulphate plug placed in the centre of the scene.

\subsection{Procedure}

In each trial, the scene was illuminated for $2 \mathrm{~s}$ : first, for $1 \mathrm{~s}$ at $25000 \mathrm{~K}$, and then for $1 \mathrm{~s}$ at $6700 \mathrm{~K}$, with no interval between. The illuminant on the test object changed either consistently or inconsistently with the scene illuminant by a variable degree quantified within the CIE $1976\left(u^{\prime}, v^{\prime}\right)$ colour space. In the consistent condition, the illuminants on the test object and on the scene were the same (both either $25000 \mathrm{~K}$ or $6700 \mathrm{~K}$ ). In the inconsistent condition, the first illuminant on the test object was the same as that on the scene $(25000 \mathrm{~K})$, but when the scene was illuminated by the second illuminant $(6700 \mathrm{~K})$, the colour of the test object could be any of 48 different colours defined by a grid with a spacing of 0.005 units in the $\left(u^{\prime}, v^{\prime}\right)$ space, as illustrated by the solid symbols in figure $2 \mathrm{a}$. The luminance of the test object remained constant. 
In each trial, observers had to decide whether the surface of the test object had changed. Although there was no time limit, observers tended to respond within the $5 \mathrm{~s}$ following offset of the stimulus. All three scenes were used with the five test objects and the order of scenes and test objects was balanced over sessions. Each observer performed about 1000 trials with each test object and scene in several sessions of $1 \mathrm{~h}$ each, during which they could take short breaks.

\subsection{Observers}

There were four observers: all except one (one of the authors, VA) were unaware of the purpose of the experiment. Each had normal colour vision as assessed by Rayleigh and Moreland anomaloscopy and the Farnsworth-Munsell 100-hue test.

\section{Results}

For each test object, a plot was obtained of the frequency of 'illuminant-change' responses as a function of the position of the test object in the CIE $1976\left(u^{\prime}, v^{\prime}\right)$ space, as shown in figure $2 \mathrm{a}$ for a yellow test object and for a single observer. The response frequency was normalised to unity at maximum. The contours show points of equal frequency. A smooth surface (not shown) was fitted to the data and its maximum determined (indicated in figure $2 \mathrm{a}$ by the open circle).
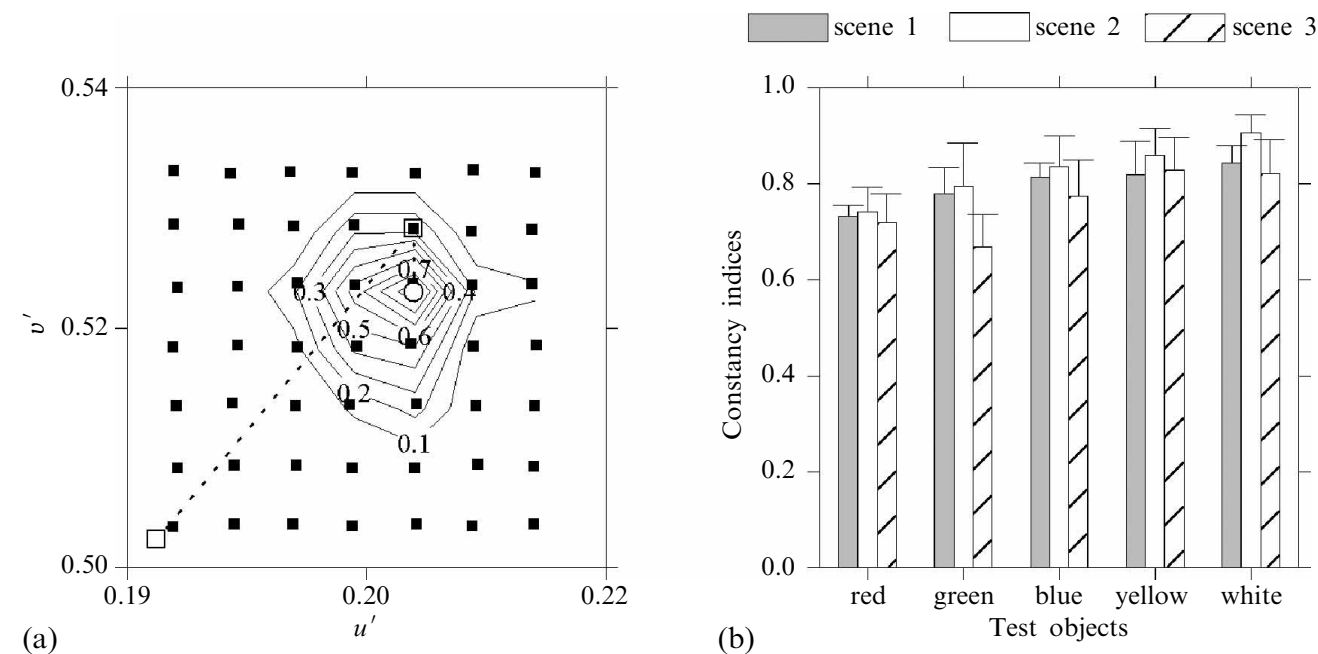

Figure 2. (a) Contour plots in the CIE $1976\left(u^{\prime}, v^{\prime}\right)$ space representing for one observer the frequency of 'illuminant-change' responses with a yellow test object. The frequency was normalised to unity at maximum. The contours show points of equal frequency. A smooth surface (not shown) was fitted to the data and its maximum determined (indicated by the open circle). Open square: coordinates of test object under $25000 \mathrm{~K}$ and $6700 \mathrm{~K}$ illuminants; solid symbols: coordinates of each point of test grid. (b) Constancy indices with five test objects and three scenes. Scene 1 had a simple uniform surround; scene 2 had a complex surround of unsaturated coloured objects; and scene 3 had a complex surround of saturated coloured objects. Indices estimated from the average data of four observers. Error bars indicate +1 SEM.

The degree of colour constancy for each test object and scene was quantified by a standard index, defined as follows (Arend et al 1991). In $\left(u^{\prime}, v^{\prime}\right)$ space, let $a$ be the Euclidean distance from the coordinates of the test object under $6700 \mathrm{~K}$ and the peak of the response distribution, and let $b$ be the Euclidean distance between the coordinates of the object under $6700 \mathrm{~K}$ and under $25000 \mathrm{~K}$; then the constancy index $c$ is given by $c=1-a / b$. A value of $c$ of 0 corresponds to perfect inconstancy and a value of 1 corresponds to perfect constancy, where the peak of the frequency distribution coincides with the coordinates of the object under the $6700 \mathrm{~K}$ illuminant. 
Figure $2 \mathrm{~b}$ shows constancy indices averaged over observers for the five test objects and three scenes. An analysis of variance showed no significant effect of scene $\left(F_{2,6}=3.6, p=0.1\right)$, test object $\left(F_{4,12}=2.1, p>0.1\right)$, or interaction between the two $\left(F_{8,24}=0.2, p>0.5\right)$. Although constancy indices varied little with the colour of the test object, the corresponding pattern of responses showed a specific shape for each colour.

\section{Discussion}

The degree of colour constancy obtained was similar in all experimental conditions. The average constancy index of 0.80 was only slightly higher than that obtained in asymmetric colour-matching measurements with Mondrian-like stimuli presented sequentially (Foster et al 2001), suggesting that three-dimensionality has limited influence on colour constancy.

More importantly, even in natural viewing conditions, the degree of colour constancy was little affected by scene structure [although mutual reflections and stray illumination may be important with some stimuli, such as the Mach card (Bloj et al 1999), they were not critical here]. This insensitivity to scene structure is reminiscent of the finding by Arend et al (1991) with Mondrian and centre-surround stimulus arrays, and by Kraft et al (2002) with more complex stimuli. It is, in particular, consistent with an interpretation of surface-colour judgments based on simple scene relationships, such as the spatial ratio of cone excitations between one surface and one or more others (Amano and Foster 2004; Nascimento and Foster 1997), or more global judgments based on minimising the variance among cone-excitation ratios (Nascimento et al 2004).

Acknowledgments. This work was supported by the Centro de Física da Universidade do Minho, Braga, Portugal, the Remote Sensing Unit, University of Beira Interior, Covilhã, Portugal, and by the Engineering and Physical Sciences Research Council, UK.

\section{References}

Almeida V M N de, Fiadeiro P T, Nascimento S M C, Foster D H, 2002 "Colour constancy under illuminant changes with 3-D and 2-D views of real scenes" Perception 31 Supplement, 135

Amano K, Foster D H, 2004 "Colour constancy under simultaneous changes in surface position and illuminant" Proceedings of the Royal Society of London, Series B $2712319-2326$

Arend L, Reeves A, 1986 "Simultaneous color constancy" Journal of the Optical Society of America A $31743-1751$

Arend L E Jr, Reeves A, Schirillo J, Goldstein R, 1991 "Simultaneous color constancy: papers with diverse Munsell values" Journal of the Optical Society of America A $8661-672$

Bloj M G, Kersten D, Hurlbert A C, 1999 "Perception of three-dimensional shape influences colour perception through mutual illumination" Nature $402877-879$

Brainard D H, 1998 "Color constancy in the nearly natural image. 2. Achromatic loci" Journal of the Optical Society of America A $15307-325$

Foster D H, 2003 "Does colour constancy exist?" Trends in Cognitive Sciences 7 439-443

Foster D H, Amano K, Nascimento S M C, 2001 "Colour constancy from temporal cues: better matches with less variability under fast illuminant changes" Vision Research 41 285-293

Kraft J M, Brainard D H, 1999 "Mechanisms of color constancy under nearly natural viewing" Proceedings of the National Academy of Sciences of the USA 96 307-312

Kraft J M, Maloney S I, Brainard D H, 2002 "Surface-illuminant ambiguity and color constancy: Effects of scene complexity and depth cues" Perception 31 247-263

Lucassen M, 1993 Quantitative Studies of Color Constancy PhD thesis, IZF-TNO-Soesterberg, The Netherlands

Nascimento S M C, Almeida V M N de, Fiadeiro P T, Foster D H, 2004 "Minimum-variance coneexcitations ratios and the limits of relational color constancy" Visual Neuroscience $21337-340$

Nascimento S M C, Foster D H, 1997 "Detecting natural changes of cone-excitation ratios in simple and complex coloured images" Proceedings of the Royal Society of London, Series B $2641395-1402$

Troost J M, 1992 "The invariance of colour perception" Irish Journal of Psychology 13 440-454 


\section{PERCEPTION}

VOLUME 342005

www.perceptionweb.com

Conditions of use. This article may be downloaded from the Perception website for personal research by members of subscribing organisations. Authors are entitled to distribute their own article (in printed form or by e-mail) to up to 50 people. This PDF may not be placed on any website (or other online distribution system) without permission of the publisher. 